\title{
Fabrication and Characterization of Polymeric Optical Waveguides Using Standard Silicon Processing Technology
}

\author{
R.L.Oliveri ${ }^{\mathrm{a}}$, A.Sciuto ${ }^{\mathrm{b}}$, S.Libertino $^{\mathrm{b}}$, G. D'Arrigo $^{\mathrm{b}}$, C.Arnone $^{\mathrm{c}}$ \\ ${ }^{a}$ Dipartimento di Fisica e Tecnologie Relative, University of Palermo, Viale delle Scienze, 90128,Palermo, Italy \\ ${ }^{b}$ CNR-IMM,sez. Catania, Str.le Primosole 50, 95121,Catania, Italy \\ ${ }^{c}$ Dipartimento di Ingegneria Elettrica, Elettronica e delle Telecomunicazioni, University of Palermo, \\ Viale delle Scienze, 90128, Palermo, Italy
}

\begin{abstract}
We report the fabrication and characterization of a rib polymeric waveguide having a thick layer of oxidized porous silicon as an innovative solution for the lower cladding. The waveguide was fabricated using standard silicon substrates and Si-based technology. The multimodal guiding structure has a polymethylmetacrylate (PMMA) core and the innovative lower cladding was obtained by thermal oxidation of a porous silicon layer. The waveguide does not have the upper cladding. Propagation loss measurements were performed at $1.48 \mu \mathrm{m}$ using the cut-back method. We obtained propagation loss of about $1.7 \mathrm{~dB} / \mathrm{cm}$, confirming the possibility to use the porous silicon oxide as the lower cladding layer, for low cost waveguide applications.
\end{abstract}

\section{INTRODUCTION}

The increased interest grown in the last years in the field of optical silicon-compatible devices has driven the research to investigate on active (light sources, modulators, detectors) and passive (waveguides, splitters) optical devices compatible with the well established silicon technology [1-5]. Many hybrid solutions have been considered so far, using both semiconductors (InP, GaAs, etc.) or dielectric materials (silica, $\mathrm{LiNbO}_{3}$, etc.). Polymers have been also considered as a very promising solution to achieve optoelectronic integration, due to their mechanical and optical properties and the compatibility with VLSI (very large scale integration) processing [6-9].

Polymeric films are generally prepared from liquid solutions. Their low cost and simple processing combined with their chemical and physical properties, easily modifiable adding doping molecules, make them more attractive than the above mentioned semiconductors or dielectrics materials for optoelectronic applications. Moreover, polymers are already used in the microelectronic industry, as there is no need of new equipments or know-how. Furthermore, polymers with different functionalities can be integrated on the same VLSI chip, offering a versatile platform.
One of the main issues for the fabrication of polymer-based waveguides is the need to have a good lower cladding, able to optically isolate the waveguide core from the Si-substrate. As Si has a refractive index $(\sim 3.5)$ much higher than that of typical polymers $(\sim 1.5-1.7)$, a thick lower cladding layer must be used to avoid light coupling with the substrate. One of the most used approaches is to grow or deposit a thick silicon oxide layer as the lower cladding. Thermally grown $\mathrm{SiO}_{2}$ has a refractive index of 1.45 , hence the layer must be at least $3 \mu \mathrm{m}$ thick if the core refractive index is, e.g., 1.6 as in the PMMA case. As a result the whole structure must be processed at high temperature for a very long time (more than 60 hours). On the other hand, thick deposited oxides suffer mechanical stresses. Another approach is to use different polymers for both the lower cladding and the core. In this case the polymers forming the different layers must be chosen not only on the basis of their optical properties, but also considering polymer materials and solvents, for each layer of core and claddings, in order to prevent damages to the lower layers. These issues strongly reduce the material choice in the waveguide fabrication.

Many papers have been proposed in the literature on both fabrication and characterization of polymeric multimode waveguides having different physical, optical, and mechanical properties, whit a variety of substrates $[10,11]$. However, so far none succeeded in proposing an optimum low cost substrate and fabrication method satisfying the applications demands for the waveguide structure, including both physical and mechanical properties.

In this work, we describe the fabrication and full characterization of rib polymeric waveguides, using standard silicon substrates and Si-based technology. We used polymethylmetacrylate (PMMA) as the core, adopting an innovative solution for the lower cladding fabrication: a porous silicon oxide layer obtained by thermal oxidation of porous silicon substrate. The use of such cladding allowed the fabrication of a structure with high refractive index difference, $\Delta \mathrm{n}$, between the core and the lower cladding layer. 


\section{EXPERIMENTAL}

We used 6 inches Czochralski grown Si wafers, heavily doped with Boron $\left(2 \times 10^{19} \mathrm{~cm}^{-3}\right)$, as the substrate. The first step for the fabrication of the proposed waveguide is the formation of the porous silicon oxide lower cladding layer. This is a two steps process: the formation of a porous silicon layer and its thermal oxidation. The porous layer is obtained by electrochemical etching of a heavily doped p-type substrate using the procedure reported elsewhere $[12,13]$. The layer porosity is defined as the ratio between the loss in weight due to the process and the weight of the silicon layer (that will be porous) before etching, in percentage $[12,13]$. A $35 \%$ porosity was chosen in order to avoid mechanical instability, presence of cracks and/or dislocations. To this purpose, a current of $1.8 \mathrm{~A}$ for $180 \mathrm{~s}$ was applied to the electrochemical cell, where the wafer was placed as anode. The cathode is a gold electrode.

A $3 \mu \mathrm{m}$ thick layer of porous $\mathrm{Si}$ of was formed. A scanning electron microscopy (SEM) image of the sample cross section is shown in Fig. 1. The good quality of both the air/porous Si and porous $\mathrm{Si} / \mathrm{Si}$ interfaces can be observed.

All the SEM images shown in this paper were obtained using a Cambridge Autoscan MK250 microscope, with the samples coated by a $10 \mathrm{~nm}$ Pt sputtered film. It should be mentioned that while the thickness of the porous larger depends on etching time, its lateral dimensions can be chosen by changing the doping concentration. A porous $\mathrm{Si}$ pocket can be formed starting from a heavily doped $\mathrm{p}^{++}$pocket, e.g. using ion implantation. Thicker porous $\mathrm{Si}$ samples, up to $6 \mu \mathrm{m}$ were prepared, increasing the erosion time up to $400 \mathrm{~s}$. The waveguide structures were fabricated on the thickest porous layers (see below).

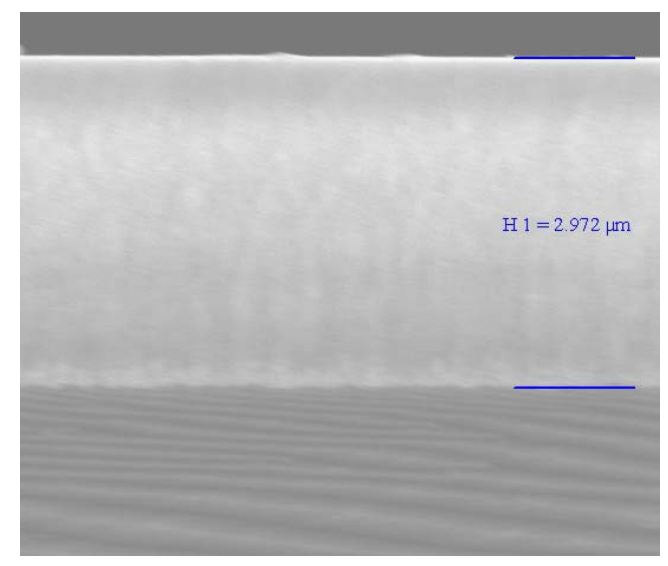

Fig. 1: SEM image of a porous Si layer. The layer thickness is $\sim 3 \mu \mathrm{m}$.
After etching, the samples underwent thermal process in $\mathrm{O}_{2}$ for 4 hours at $1100^{\circ} \mathrm{C}$, in order to ensure the oxidation of the entire porous structure. The final $\mathrm{SiO}_{2}$ layer thickness is roughly the same of the starting porous layer.

The polymeric solution used in this study was prepared dissolving PMMA $(980.000 \mathrm{~g} / \mathrm{mol})$ in Toluene, by stirring. Then, a small amount of solution was dispensed on the substrate. The samples $\left(4 \times 4 \mathrm{~cm}^{2}\right)$ were previously rinsed using a standard procedure and dried under a $\mathrm{N}_{2}$ stream. The conventional processing for the fabrication of polymeric optical waveguides includes the spin-coating of a solution of PMMA on the surface of the lower cladding to obtain a thin and uniform film. The film thickness is defined by both the rotational velocity and the solution density. Polymeric thin films were prepared using different PMMA concentrations in toluene solution and different spin speeds. The PMMA concentrations varied between $5 \mathrm{w} \%$ and $15 \mathrm{w} \%$ while the spin speed ranged from 2000 to $6000 \mathrm{rpm}$. The spinning time was fixed at $60 \mathrm{~s}$.

After spinning, the samples were baked on hot plate at $170^{\circ} \mathrm{C}$ for $90 \mathrm{~s}$, to evaporate the residual solvent. The films thickness as a function of spinning speed was studied by ellipsometry and film thickness probe (FTP) using a FTP500 Sentech Instruments $\mathrm{GmbH}$, optical profilometry and focused ion beam (FIB).

After spinning and baking, the waveguide patterns on the PMMA film were defined. An AZ1518 photoresist film was spin-coated onto the PMMA layer in order to obtain a $2.5 \mu \mathrm{m}$ photoresist film thickness. The samples were soft baked at 100 ${ }^{\circ} \mathrm{C}$ for $60 \mathrm{~s}$. The channel waveguide masks were patterned by laser direct writing photolithography using a He-Cd laser beam (DWL 66 Heidelberg Instrument) at $442 \mathrm{~nm}$ wavelength.

The guiding structures were formed by oxygen reactive ion etching (RIE) [14-20], in a Sirius SI 591 plasma etcher. The RIE was carried out at a constant $\mathrm{O}_{2}$ flow rate of $15 \mathrm{sccm}$ with total pressure of 200mTorr and power of $40 \mathrm{~W}$.

The surface roughness of both the as spun PMMA and after RIE were measured by Atomic Force Microscopy (AFM). Measurements were carried out with an AFM Digital Instruments Dimension 3100 and performed in Tapping Mode. In such mode a reduced pressure is applied on the sample, avoiding surface modification of the organic sample during the measure.

The waveguide optical characterization in the near infrared (NIR) spectral region was performed using $1.48 \mu \mathrm{m}$ singlemode pigtailed Fabry-Pérot laser diode (CQF858 JDS Uniphase) coupled with a single-mode tapered (angular opening of approximately $50^{\circ}$ ) and lensed (convergent $12 \mu \mathrm{m}$ diameter lens) fiber. It has a core diameter of $10 \mu \mathrm{m}$ but, thanks to both the taper and the lens, it provides an optical beam of $\sim 6 \mu \mathrm{m}$.

The transmitted optical signal was collected by a multimodal fiber and analyzed by an ANDO AQ-6315B spectrum analyzer working in power meter configuration. The waveguide propagation loss was measured using the cut-back method. 


\section{RESULTS AND DISCUSSION}

In order to obtain a good quality waveguide, the first important step is the formation of a good quality lower cladding, in our case a thick porous oxide layer. This leads, as mentioned in the introduction, to a processing time of about 15 hours to grow $1 \mu \mathrm{m}$ thick thermal silicon oxide layer. Another important characteristic of the porous $\mathrm{SiO}_{2}$ is its refractive index. In fact, since the $\mathrm{SiO}_{2}$ is porous, its refractive index (n) is lower than 1.45, the thermally grown $\mathrm{SiO}_{2} \mathrm{n}$ value. From the layer porosity we evaluated a value of 1.3 for the real part of the refractive index. As a result, only an about $2 \mu \mathrm{m}$ thick porous layer would be needed to assure a good vertical optical confinement. Nevertheless, we used the thickest oxide $(\sim 6 \mu \mathrm{m}$ thick) in order to check the layer mechanical strength and its resistance to all the waveguide fabrication steps. The oxide quality was tested by ellipsometry and the oxide refractive index value is in agreement with the calculated one.

The second important point addressed during this work was the determination of the parameters to obtain a fixed PMMAbased core thickness, with good optical quality. From the film thickness measurements we found that the PMMA thickness decreases following roughly the relation,

$$
h \approx f^{-\lambda}
$$

where $h$ is film thickness, $f$ is spinning speed and $0.43 \leq \lambda \leq 0.49$. This result does not hold for photoresist films whose thickness is proportional to the inverse of the spinning speed. If the solution viscosity remains constant during spinning, theoretical studies show $h \propto 1 / f$, as in the photoresist case [8,14]. On the other hand, eq. (1), is often observed when the polymeric solution solvent evaporates during the spin-coating process. As a result, the solution viscosity changes during film deposition [15-17]. Our FTP measurements indicate a thickness uniformity within $15 \%$. The measurements were carried out on at least 5 points, taking more measurements for each point.

A fundamental parameter to evaluate fabricating polymeric layers for waveguide applications is the surface roughness and the quality of the interface between the substrate (porous silicon oxide in our case) and the deposited film. To this purpose, AFM measurements were carried out and the results on the asprepared films are shown in Fig. 2. The measured roughness mean square value is $0.31 \mathrm{~nm}(\mathrm{rms})$.

Finally, ellipsometry measurements carried out on the spinned PMMA film provide a refractive index value of about 1.55 at a probe wavelength of $633 \mathrm{~nm}$, in agreement with literature data. This result confirms the good optical quality of the deposited films, indicating the possibility to fabricate light waveguides using such films as the core. Low optical loss is essential for optical waveguide devices and it is strictly related to the fabrication processes, responsible of both the bulk and the film surface qualities. In waveguides fabricated by plasma etching, light scattering is essentially due to roughness of the etched surface and side-walls.

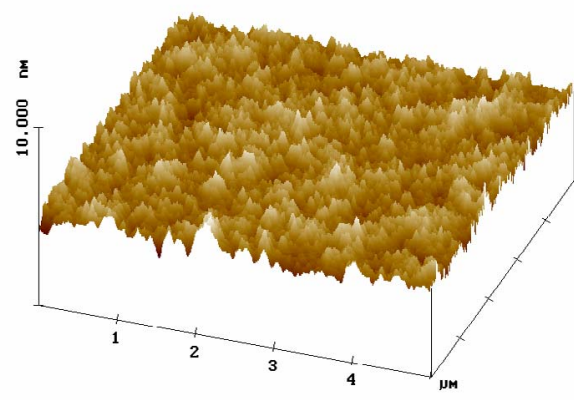

Fig. 2: AFM surface image for a sample area of $25 \mu \mathrm{m}^{2}$ of the spin-coated PMMA film: the roughness is represented in a grey scale referred to the vertical scale of $10 \mathrm{~nm}$. Darker grey indicates the lower height.

In RIE, several parameters such as rf power, gas flow and chamber pressure can be adjusted in order to minimize the final surface roughness. Therefore, the optimization of RIE process is critical to obtain a smooth etched surface and side-wall with low roughness [18-20]. In fact, both the etch rate and the rms roughness increase when increasing the applied $\mathrm{rf}$ power. As a final result, a significant increase in the scattering loss is obtained in the optical waveguide. Moreover, the etch rate and rms surface roughness increase with the chamber pressure up to 100 mTorr, while both decrease with a further pressure increase. Hence, we expected that low rf power and high chamber pressure could produce smoother film surfaces. To this purpose the etching parameters where studied and the final film surface quality was tested by AFM.

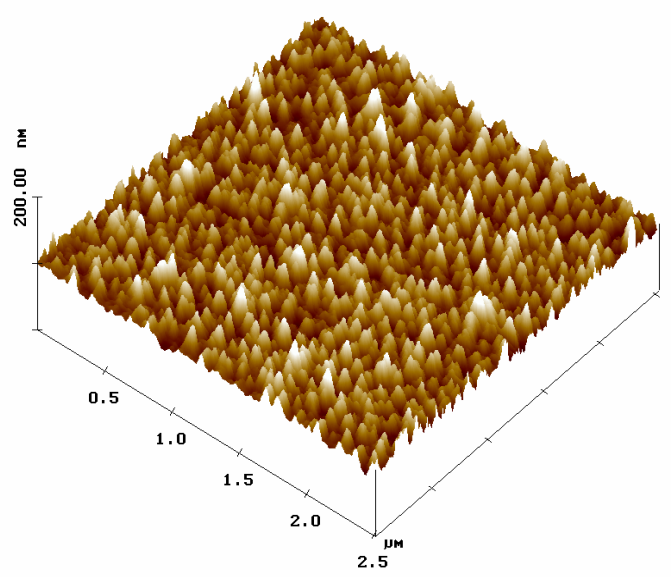

Fig. 3: AFM surface image of sample of PMMA film after a 12 min etching process at $200 \mathrm{mTorr}, 40 \mathrm{~W}$ rf power, $15 \mathrm{sccm} \mathrm{O}$ flux at. The image refers to an area of $6.25 \mu \mathrm{m}^{2}$ with a vertical scale of $100 \mathrm{~nm}$. Darker gray indicates regions at lower height. 
As an example, the AFM surface image of the sample after 12 min etching at $200 \mathrm{mTorr}, 40 \mathrm{~W}$ rf power, $15 \mathrm{sccm} \mathrm{O}$ flux, is shown in Fig. 3. Also in this case the grey scale indicate the roughness. The surface roughness is shown using a vertical scale of $100 \mathrm{~nm}$ on a sample area of $6.25 \mu \mathrm{m}^{2}$. Its value is of about $9.6 \mathrm{~nm}$ (rms). After the RIE process the surface roughness has remarkably increased, from $0.31 \mathrm{~nm}$ (bulk film value, see Fig. 2) up to 9.6, after 12 minutes etching. An increase in the etching time will cause a further increase in the surface roughness (see below). The final etching parameters used in this work were chosen in order to minimize the PMMA surface roughness and their values are in agreement with literature data [18]. An important aspect we considered in RIE parameters optimization was the determination of the ratio between the PMMA and the photoresist etching rate. During the process, both PMMA and photoresist are etched, as the fabrication method is based on pattern transfer from the patterned photoresist to the PMMA film underneath.

In order to study the etching rate of the photoresist and the PMMA we measured the resulting thickness of both layers as a function of the etching time (Fig. 4). All measurements were made on samples previously hard-baked at $120^{\circ} \mathrm{C}$ for $50 \mathrm{~min}$. The linear fits of the experimental data provide etching rates of $206 \pm 5 \mathrm{~nm} / \mathrm{min}$ and $164 \pm 2 \mathrm{~nm} / \mathrm{min}$ for PMMA and photoresist respectively.

As mentioned before, the polymer etching process, needed for the definition of the waveguide core, is one of the most critical steps as the scattering losses are directly related to the quality of the waveguide walls.

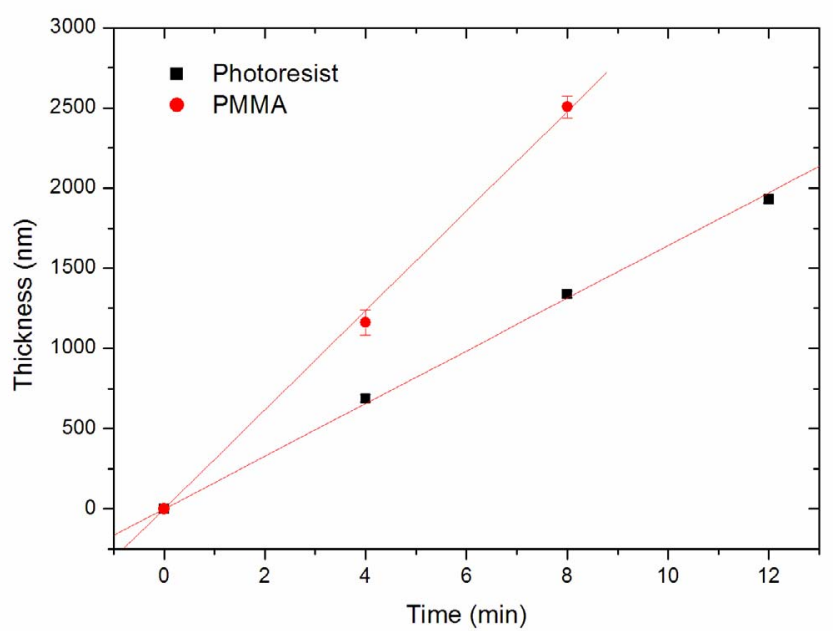

Fig. 4: Final thickness versus the RIE etching time for PMMA (circles) and photoresist (squares).

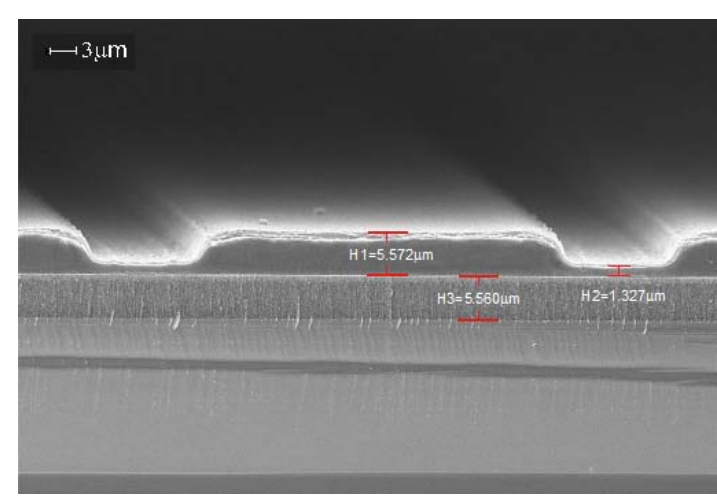

Fig. 5: SEM image of a PMMA waveguide cross section. The ruler (upper left) as $3 \mu \mathrm{m}$ wide.

The waveguide structural quality was studied by SEM images. An example a SEM cross is shown in Fig. 5.

The porous oxide is flat and uniform and it is possible to observe the absence of structural defects due to mechanical stress at the oxide interfaces with the PMMA film and with the silicon substrate. This result confirms the good quality of the fabricated oxide layer.

The waveguide rib is $50 \mu \mathrm{m}$ wide, laterally defined by $5 \mu \mathrm{m}$ wide trenches. A residual PMMA layer, $1.3 \mu \mathrm{m}$ thick, can be observed at the bottom of the trenches. In the same figure, the lower cladding of porous silicon oxide is clearly visible and the measure indicates a thickness of $5.6 \mu \mathrm{m}$. Moreover, the structural analysis provides a rib height of about $4.2 \mu \mathrm{m}$, given by the difference between the film thickness and the residual PMMA thickness in the trenches. Such value is greater than the $3 \mu \mathrm{m}$ value expected from the measured etching rate and the used processing parameters. In particular, the PMMA was overetched by $400 \mathrm{~nm}$. This discrepancy is due to the method used for the etching rate measurements. In fact, the data reported in Fig. 4 were taken on the same sample that underwent subsequent incremental etching times, while the waveguide was fabricated in a single etching step lasting $16 \mathrm{~min}$.

Fig. 5 also shows that the sidewalls are not perfectly vertical. In fact, even if the RIE is a strongly anisotropic etching, there is not a hard mask to shield the PMMA, and the photoresist is progressively eroded where exposed to the plasma. The smoothing is also due to a relaxation of the structure during photoresist post-bake before the etching process.

An optimization of the thermal processes the polymer has to go through is desirable in order to avoid undesired effects such as the non verticality of the rib walls and the light scattering due to the roughness of the sidewalls. 


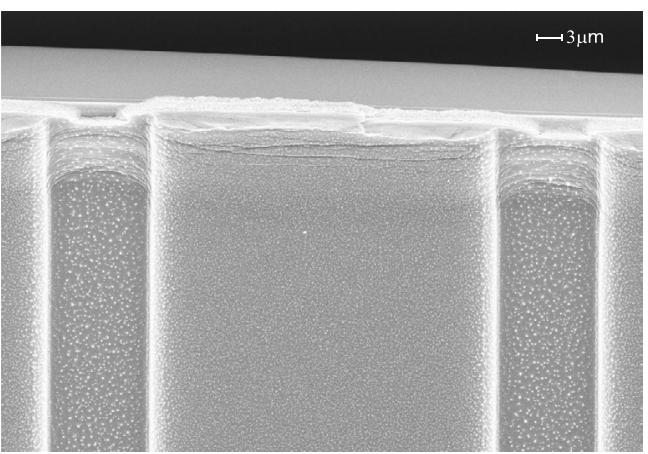

Fig. 6: SEM top view of a PMMA waveguide.

The waveguide quality has also been checked, by observing, the sample surface from top. A typical image is shown in Fig. 6. Several defects and cracks are visible close to the cleaved side. They formed during cleavage. Moreover, it can be observed that the roughness (small white dots in figure) in the trenches is higher than on top of the waveguide rib.

As mentioned before the etching process used to fabricate the waveguide was $16 \mathrm{~min}$ long. The measured roughness after etching was measured by AFM and is reported in Fig. 7 for a sampling area of $100 \mu^{2}$. It should be noticed that the vertical scale is $500 \mathrm{~nm}$, much larger than the scales of Fig. 2 and 3. The measure provides a roughness of about $31.2 \mathrm{~nm}(\mathrm{rms})$. This measurement confirms that the film roughness increases with the etching time. In particular, it increases from $0.31 \mathrm{~nm}$ in the as prepared sample to $9.6 \mathrm{~nm}$ after 12 min etching and reaches $31 \mathrm{~nm}$ after $16 \mathrm{~min}$ etch. Of course, the waveguide quality, in terms of scattering losses, is strongly affected by roughness.

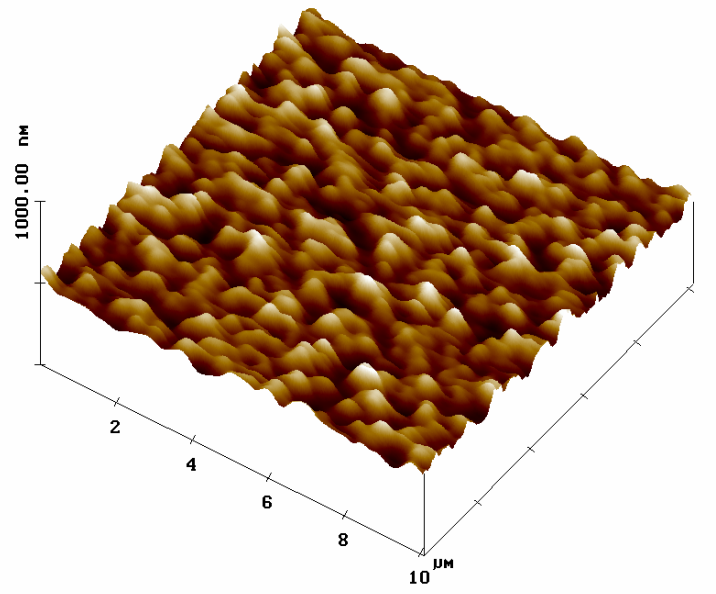

Fig. 6: AFM of the rib top (16 min etching process at $200 \mathrm{mTorr}, 40 \mathrm{~W}$ rf power, $15 \mathrm{sccm} \mathrm{O}_{2}$ flux). The measurement was carried out in an area of $100 \mu \mathrm{m}^{2}$ and exhibits a vertical scale of $500 \mathrm{~nm}$. Darker regions are deeper.
The observed increase in the surface roughness is probably due to the presence of solvent in the photoresist layer. It etched the PMMA film surface even before the RIE. The roughness at the interface between the photoresist and the PMMA layer has bee subsequently transferred to the waveguide. The roughness on the rib top is lower than in the other waveguide surface regions, due to shorter etching time.

In order to perform the optical characterization, through light injection into the waveguide and collection at its end, the wafer was cleaved, to provide waveguide end faces for fiber coupling. The cleavage procedure has the effect to increase the optical loss due to the formation of the defects already observed in Fig. 6 , hence the obtained values are an upper limit to the propagation losses. These were measured using the cut-back method.

From the transmitted intensity and the optical input power, the total attenuation was calculated. The output intensity was measured after consecutive cleavages of the same waveguides. The experimental data are summarized in Fig. 8. The linear fit of the data allowed us to calculate the total loss (coupling and transmission loss). It should be pointed out that the fiber coupling conditions were the same during all measurements.

From the fit slope we estimated a propagation loss in the waveguide of about $1.7 \mathrm{~dB} / \mathrm{cm}$. The linear fit intercept with the vertical axis, provides an estimated coupling loss of about $9 \mathrm{~dB}$. The obtained propagation loss is approximately twice than that reported on the literature, $(0.83 \mathrm{~dB} / \mathrm{cm}$ at $\lambda=1.55 \mu \mathrm{m})$ for similar structures having thick silicon oxide as lower cladding or polymeric lower cladding [21]. But one result is very promising, as it demonstrates that it is possible to use porous silicon oxide as the lower cladding.

The high coupling loss as measured is due the quality of the guide end faces: the samples were just cleaved, with no additional polishing to improve the planarity and orthogonality of the waveguide end faces.

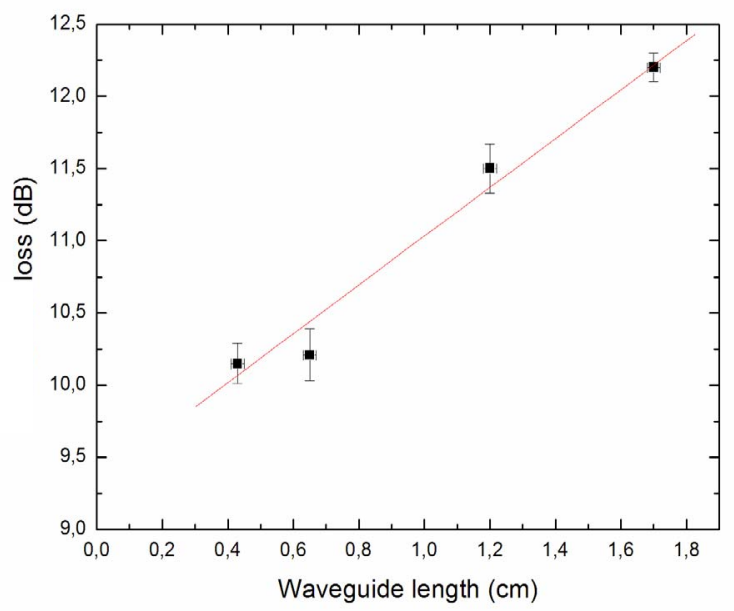

Fig. 7: Optical loss dependence of the fabricated waveguide on the waveguide length at $1.48 \mathrm{um}$ 


\section{CONCLUSION}

We fabricated passive waveguides having PMMA as the core and a thick layer porous silicon oxide (up to $6 \mu \mathrm{m}$ ) as the lower cladding. The waveguides were not covered with an upper cladding. The results demonstrate that the porous oxide layer is uniform and has no crack or dislocation. Moreover, there is no evidence of mechanical stress at the interfaces with the silicon substrate and with the polymeric core film, even after the full processing. The propagation loss, is about $1.7 \mathrm{~dB} / \mathrm{cm}$ at $\lambda=1.48 \mu \mathrm{m}$. Lower losses could be achieved by a more careful control of the RIE process, that would minimize the surface roughness as well as the side-wall roughness. Our results since confirm the possibility to use a cladding layer made of the innovative porous silicon oxide, in order to decrease the waveguide fabrication cost. We are also confident that such cladding is a valid alternative whenever it is necessary to use a sturdy and flexible substrate. Moreover, the low thermal budget needed to fabricate the thick oxide may be really important if the waveguide process has to be integrated in a device fabrication flow-chart. It is also useful when heat sensitive elements, such as certain optical non-linear materials or crystals, have to be embedded in the film.

\section{ACKNOWLEDGEMENTS}

The authors would like to acknowledge S. Di Franco, F. Iacona, A. La Mantia, F. Giannazzo for their technical and scientific assistance, F. Roccaforte, G. Fontana for their helpful suggestions.

\section{REFERENCES}

[1] N.M.Jokerst, M.A.Brooke, S.-Y.Cho, et al. "The Heterogeneous Integration of Optical Interconnections Into Integrated Microsystems" IEEE J. Selected Topics in Quantum Electronics, vol. 9 (2), pag. 350 (2003).

[2] E.Suhir, "Microelectronics and Photonics-The future", Microelectronics Journal, vol. 31, pag. 839 (2000).

[3] A.V. Mulè, E.N. Glytsis, T.K. Gaylord, J.D. Meindl, "Electrical and Optical Clock Distribution Networks for Gigascale Microprocessors", IEEE Transactions on VLSI Systems,vol. 10 (5), pag. 582 (2002).

[4] M. Bruel, "Silicon on Insulator Material Technology", Electronics Letters, vol. 31 (14), pag. 1201 (1995).

[5] U.Fischer, T.Zinke, J-R.Kropp, F.Arndt, K.Petermann, " $0.1 \mathrm{~dB} / \mathrm{cm}$ Waveguide Losses in Singlemode SOI Rib Waveguides", IEEE Photonics Technology Letters, vol. 8 (5), pag. 647 (1996).

[6] L.R.Dalton, A.Harper, A.Ren, F.wang, G.Todorova, J.Chen, C.Zhang, M.Lee "Polymeric Electro-optic Modulators: From Chromophore Design to Integration with Semiconductor VLSI Electronics and Silica Fiber Optics", Industrial \& Engineering Chemistry Research, vol. 38, pag. 8 (1999).

[7] H.Ma, A.K.-Y.Jen, L.R.Dalton "Polymer-Based Optical Waveguides: Materials, Processing and Devices", Advances Materials,vol. 14 (19), pag. 1339 (2002).

[8] M.Hikita, R.Yoshimura, M.Usui, S.Tomaru, S.Imamura, "Polymeric Optical Waveguides for Optical Interconnections", Thin Solid Films, vol. 331, pag. 303 (1998)
[9] L.Eldada, L.W.Shacklette, "Advances in Polymer Integrated Optics", IEEE J.of Selected Topics in Quantum Electronics, vol. 60 (1), pag. 5 (2000).

[10] M. Kagami, H. Ito, T. Ichikawa, S. Kato, M. Matsuda, N. Takahashi, "Fabrication of large-core high-D optical waveguides in polymers", Appl. Optics, vol. 34 (6), pag. 1041 (1995).

[11] S.Imamura, R.Yoshimura, and T.Izawa, "Polymer channel waveguides with low loss at $1.3 \mu \mathrm{m}$ ”, Electron. Lett. 27, pag. 1342 (1991).

[12] R.L.Smith, S.D. Collins, "Porous silicon formation mechanisms", J.of Apl. Phys. 71 (8), R1 (1992).

[13] Properties of porous silicon, ed. by L. T. Canham, DERA, Malvern, UK, IEE Inspec (1997).

[14] S.Nonogaki, T.Ueno, T.Ito, "Microlithography Fundamentals in Semiconductor Devices and Fabrication Technology", Marcel Dekker Inc.(1998).

[15] D.Meyerhofer, "Characteristics of Resist Films Produced by Spinning", J. Appl. Phys. vol. 49, pag. 3993 (1978).

[16] D.Bornside, C.Macasko, L.Scriven, "Spin Coating: One-Dimensional Model”, J.Appl.Phys. vol. 66, pag. 5185 (1989).

[17] W.W.Flack, D.S.Soong, A.T.Bell, D.W.Hess, "A Mathematical Model for Spin Coating of Polymer Resist”, J.Appl.Phys., vol. 56 (4), pag. 1199 (1984)

[18] J.W.Kang, J.S.Kim, J.J.Kim, “Optimized Oxygen Plasme Etching of Polycarbonate for Low-Loss Optical Waveguide Fabrication” Japan J. Appl. Phys. Vol. 40, pag. 3215 (2001).

[19] N.Agarwar, S.Ponoth, J.Plawsky, P.D.Persans, "Roughness Evolution in Polyimide Films Durino Plasme Etching”, Appl. Phys. Lett. vol. 78 (16), pag. 2294 (2001).

[20] O.Joubert, J.Pelletier, Y.Arnal, "The Etching of Polymers in OxygenBased Plasma: A Parametric Study”, J. Appl. Phys. vol. 65 (12), pag. 5096 (1989).

[21] R.Yoshimura, M.Hikita, S.Tomaru, S.Imamura, "Low-loss Polymeric Optical Waveguides Fabricated with Deuterated Polyfluoromethacrylate", J.Lightwave Technology, vol. 16 (6), pag. 1030 (1998). 